\title{
Treatment Outcomes and Associated Factors in Hospitalised Children with Severe Acute Malnutrition: A Prospective Cohort Study
}

This article was published in the following Dove Press journal: Pediatric Health, Medicine and Therapeutics

\author{
Fuad Adem' \\ Dumessa Edessa (DI \\ Bodena Bayissa $\mathbb{D}^{2}$ \\ Mesud Mohammed Hassen ${ }^{3}$ \\ Mohammed A Mohammed ${ }^{4}$ \\ 'Department of Clinical Pharmacy, \\ School of Pharmacy, Haramaya \\ University, Oromia, Ethiopia; ${ }^{2}$ Jimma \\ University, School of Pharmacy, Jimma, \\ Ethiopia; ${ }^{3}$ Madda-Walabu University, \\ School of Medicine and Health Sciences, \\ Madda-Walabu, Ethiopia; ${ }^{4}$ University of \\ Auckland, School of Pharmacy, Auckland, \\ New Zealand
}

Correspondence: Fuad Adem Email Fuadamoh2017@gmail.com
Background: Malnutrition continues to be a public health challenge in sub-Saharan African countries. In Ethiopia, there is a paucity of data regarding factors affecting treatment outcomes in children with severe acute malnutrition (SAM).

Methods: A prospective cohort study was conducted among children aged 6 to 59 months with SAM, receiving care at Jimma University Medical center, Ethiopia. Bivariate and multivariate analyses were computed to determine factors associated with treatment outcomes. Kaplan-Meier survival analysis, life-table analysis, and Log rank test were used to determine death rates, estimate the proportion of surviving, and compare time to recovery (nutritional cure).

Results: A total of 133 children were included in this study and $79.7 \%$ had medical comorbidities. Overall, nutritional recovery, death, and default rates were $25.6 \%, 3.8 \%$, and $7.6 \%$, respectively. There was no significant difference in the nutritional recovery rate ( $26.1 \%$ versus $25.4 \%$; $=0.4$ ) and the median time to recovery between children who had diarrhea at admission (26 days; 95\% CI: 24.0-28.7) and those who had not (26.0 days; 95\% CI: 21.90-30.10). Likewise, the average daily weight gain was not significantly different between the two groups $(6.34 \mathrm{~g} / \mathrm{kg} /$ day versus $7.76 \mathrm{~g} / \mathrm{kg} /$ day, $\mathrm{p}=0.4)$. Having diagnosed with tuberculosis (Adjusted Hazard Ratio $(\mathrm{AHR})=0.19$, CI 0.06-0.62) and anemia (AHR $=0.32$, CI $0.14-0.74)$ and treatment failures $(\mathrm{AHR}=0.17, \mathrm{CI}, 0.16-0.03)$ were predicting factors for time to recovery.

Conclusion: The recovery rate and average daily weight gain were found to be sub-optimal in the study population. However, the median time to recovery was within the national recommendation. There was no significant difference in the recovery rate and time to recovery between the two groups. Treatment failures and the presence of tuberculosis and anemia were indicators for prolonged stabilization phase and time to achieve nutritional cure. Optimal average daily weight gain and clinical management of comorbidities may enhance early recovery in hospitalised children with SAM.

Keywords: severe acute malnutrition, treatment outcome, survival status, hospital-based care, prospective cohort

\section{Introduction}

Malnutrition is a state in which the nutritional status of person is suboptimal and thereby health and growth may be impaired. It may be caused by an illness that impairs nutritional intake and metabolism, inadequate intake of macronutrients/ micronutrients. ${ }^{1,2}$ Acute malnutrition or wasting is a form of undernutrition which is defined as low weight for height (WFH) or the presence of nutritional edema. ${ }^{1}$ 
Acute malnutrition (AM) can be moderate acute malnutrition (MAM) or severe acute malnutrition (SAM) based on its severity. Worldwide, acute malnutrition affects about $8 \%$ (52 million) of under-five children and it is responsible for about $4.4 \%$ of nutrition-related under-five mortality. ${ }^{2,3}$

SAM is a nutritional emergency characterized by the presence of weight-for-height (WFH) $Z$ score $<-3$, midupper arm circumference (MUAC) $<11.5 \mathrm{~cm}$ and bilateral nutritional pedal edema. ${ }^{3}$ Globally, SAM affects more than 17 million children causing 2.9 million hospitalisations out of which about $17 \%$ of them requiring treatment. ${ }^{2,4}$ In Africa, 14 million children under the age of 5 years have acute malnutrition, out of this, SAM accounts for about 4.1 million cases. $^{2}$

In Ethiopia, undernutrition continues to be a major public health problem accounting for more than $50 \%$ of under-five mortality. ${ }^{5}$ According to the Ethiopian Demographic and Health Survey (EDHS) data, ${ }^{6}$ there has been some reduction in the prevalence of chronic malnutrition (stunting) over the past two decades (ie, $57.8 \%$ in $2000,44.4 \%$ in 2011, and $40 \%$ in 2014). Similarly, a modest reduction in the prevalence of AM has been observed (ie, $12.5 \%$ in $2000,9.7 \%$ in 2011 , and $9.0 \%$ in 2014). However, between the years 2014 and 2016, although there has been a further reduction in the prevalence of chronic malnutrition (stunting) from $40 \%$ to $38 \%$, a slight increase (from $9 \%$ to $10 \%$ ) in the prevalence of AM has been observed. ${ }^{7}$ Previous research studies related to SAM have been focused on analysis of secondary data. Thus, there is a paucity of data from prospective studies regarding factors associated with treatment outcomes in hospitalised children with SAM. Moreover, no such studies have been conducted at Jimma University Medical center, southwest Ethiopia. Therefore, evaluation of nutritional recovery rate and its associated factors in under-five children with SAM receiving care at Jimma University Medical Center (JUMC), Nutritional Rehabilitation Unit (NRU) was deemed vital to generate data that inform evidence-based clinical decision-making to optimize the care of children.

\section{Methods}

\section{Study Design and Setting}

A prospective cohort study was conducted to assess treatment outcomes and associated factors in children with SAM receiving care at Jimma University Medical center, Ethiopia. Jimma University Medical Center is a teaching and referral hospital with 600-bed capacity in southwest Ethiopia. The hospital yearly provides services for 218,095 outpatients, 166,778 inpatients, 14,207 emergencies and 5973 deliveries from the catchment population of about 15-20 million.

\section{Selection of Study Subjects}

The study subjects for this study were children with SAM who were admitted to the hospital from March to July 2018. Consecutive sampling method was used to select the children for the study. The inclusion criteria we considered involved children/infants aged between 6 and 59 months, children/infants with $\mathrm{WFH}<-3 \mathrm{Z}$ score or MUAC $<11.5 \mathrm{~cm}$ with length $>65 \mathrm{~cm}$ at admission, and children who had bilateral nutritional pitting edema with any complication. Infants/children whose parents/guardians decline to consent their child's participation in the study were excluded. The study subjects were divided into two groups based on the presence or absence of diarrhea at hospitalisation, to see the effect of having or not having diarrhea at admission in treating children hospitalised with SAM.

\section{Data Collection Process}

The data were collected using a structured data collection format that consists of sociodemographic variables, nutritional status, anthropometric measurements, laboratory tests, and medical conditions at admission and medical treatment during the study period. Data collection instrument was reviewed and enhanced by one clinical pharmacist and one pediatric resident. The data were collected by four medical interns and two clinical nurses after being given a training on the data collection procedure. The data collectors visited the pediatric ward daily and assessed all the newly admitted children's eligibility for inclusion into the study based on the predefined inclusion criteria. Sociodemographic data and baseline clinical information, vital signs, anthropometry measurements, nutritional and other medical diagnoses, laboratory results, and treatments were key variables collected by this study at baseline and during follow-up period. Accordingly, weight and edema secondary to malnutrition were documented daily when presented. At admission, all children were examined by attending physician and clinically evaluated to identify the presence of medical comorbidities. Complete blood count (CBC) to identify anemia and/or other medical problems, plasma glucose level and blood film for malaria parasites, Gene-Xpert/RIF test for mycobacterium tuberculosis, and HIV tests were done at admission. Stool examination and 
urine analyses were done to assess any evidence for the presence of parasites and urinary tract infections, respectively.

During the follow-up, experiences of new symptoms such as vomiting, diarrhea, and breathing difficulty were assessed. Besides, the data collectors monitored child feeding using 24-hour dietary recall methods until the date of discharge, or day 40 of therapeutic feeding. Finally, treatment outcome at discharge or day 40 of the admission and time to event were recorded.

\section{Definitions and Measurements}

In this study, SAM was defined by the presence of bilateral pitting edema or severe wasting (ie, MUAC $<11.50 \mathrm{~cm}$ or WFH $<-3 Z$ score). ${ }^{8}$ WFH was measured using the WHO growth standards for children under 5 years of age. ${ }^{9}$ Nutritional recovery or cure was considered when a child met the discharge criteria for inpatient therapeutic feeding program (MUAC $\geq 12.5 \mathrm{~cm}, \mathrm{WFH} \geq-2 \mathrm{Z}$ score and have no edema for at least two weeks). The Sphere Project that sets standards for an outcome measure of children hospitalised with SAM was also considered. A child for whom two consecutive weighing (ie, for 2 days) were absent during inpatient therapeutic feeding program was considered defaulter. ${ }^{10}$ If a child died while receiving care in the facility, the clinical condition was recorded as death. ${ }^{10} \mathrm{On}$ the contrary, if a child's clinical information was recorded as death, defaulter, transfer out or unknown status, then this was operationally defined as censored. Weight gain was calculated using the formula provided in the national guideline for inpatient management of children with SAM. That means a difference of discharge weight and admission weight in grams was divided by admission weight in kilograms and duration of hospital stay in days. Average weight gain was considered as a sum of weight gain divided by the number of children who were recovered nutritionally.

\section{Data Processing and Analysis}

The collected data were cleaned, coded, and entered into Statistical Package for Social Sciences (SPSS) for Windows version 21.0 (SPSS Inc., Chicago, IL, USA). Descriptive analysis was performed using proportion for categorical data and mean or median for continuous variables. Pearson chi-square test was used to see an association between categorical variables while the independent two-sample $t$-test was used to compare means between groups. Treatment outcomes were categorized into censored and recovered, and nutritional recovery was considered as a dependent outcome variable. The Kaplan-Meier survival analysis, life-table analysis, and Log rank test were used to determine a rate of nutritional recovery over time, to estimate proportion surviving within each interval and to compare time to recovery, respectively. Bivariate then multivariate regression analyses were used to identify predictors of treatment outcomes (nutritional recovery or cure). Statistical significance was determined by using P-values $<0.05$ and $95 \%$ confidence intervals.

\section{Ethical Consideration}

Ethical approval for the study was obtained from the Institutional Review Board of Jimma University (reference number IHR PGS/213/18). Parents and caregivers of the children participating in the study were provided a brief description about the benefits and risks of the study, and how authors maintain confidentiality of any identifying information on health records of children included in the study. Informed, voluntary and written consent was obtained from family or caregivers of each child before commencing the data collection.

\section{Results}

\section{Sociodemographic Characteristics and Anthropometric and Laboratory Measurements}

From 892 children admitted to the hospital during the study period, 179 were diagnosed with severe acute malnutrition. After excluding twenty-nine children aged less than six months and seventeen children aged greater than 59 months, 133 children met the inclusion criteria. From the total of 133 children, $68(51.1 \%)$ were female and most $77(59.9 \%)$ of them were aged less than 24 months and were from rural areas $(n=112,84.2 \%)$. More than half $(n=69,51.9 \%)$ of the children had diarrhea at admission due to complication of SAM.

The mean weight at admission was $8.8( \pm 4.7) \mathrm{kg}$ for children who had diarrhea and $11.5( \pm 7.01) \mathrm{kg}$ for those children who had not diarrhea. The mean weight at admission was not significantly different between the two groups $(p=0.011)$. Likewise, the mean length/height at admission was not significantly different between children who had diarrhea at admission and children who had not diarrhea at admission. 
The mean MUAC at admission was $11.50( \pm 1.41) \mathrm{cm}$, and $11.10( \pm 1.61) \mathrm{cm}$ for children presenting with diarrhea and those without, respectively $(\mathrm{p}=0.154)$

The majority of the children (80.5\%) had MUAC below $11.5 \mathrm{~cm}$ and $42.1 \%$ of them had WFH less than $70 \%(-3 \mathrm{SD})$ at admission. At admission, $37.6 \%$ of the children had both low mean MUAC $(<11.5 \mathrm{~cm})$ and low WFH $(<-3 \mathrm{SD})$.

CBC test results were available for 99 (74.4\%) children. The mean (SD) hemoglobin level at admission was $9.1 \mathrm{~g} / \mathrm{dl}( \pm 3.1 \mathrm{~g} / \mathrm{dl})$ for children presenting with diarrhea and $9.5 \mathrm{~g} / \mathrm{dl}( \pm 2.7 \mathrm{~g} / \mathrm{dl})$ for those without. Gene-Xpert/RIF test revealed diagnosis of tuberculosis in eight children. Again, five children had hypoglycemia with random blood glucose level $<54 \mathrm{mg} / \mathrm{dl}$ and three children were blood film tested positive for malaria. HIV status was known for $53(39.85 \%)$ children and 50 of them had negative test results (Table 1). The overall mean laboratory test results were not significantly different between children who had diarrhea at admission and those who had not.

\section{Medical Comorbidities}

Of the total 133 children, $79.7 \%$ had at least one documented comorbid medical condition. Fourteen (20.3\%) children with diarrhea and $9(14.0 \%)$ children without diarrhea at admission had Kwash-dermatosis. Again, 27 (39.1\%) children with diarrhea and 27 (37.7\%) children without diarrhea had anemia (Table 2)

\section{Medication and Therapeutic Feedings}

All the 133 children were given formula 75 (F-75) that contains 75 kilocalories per 100 milliliters. Again, 115 (86.5\%) children (ie, 60 from those children with diarrhea at admission and 55 from those without) were prescribed formula 100 (F-100) that contains 100 kilocalories per 100 milliliters while fifty-three children (ie, 27 (39.1\%) from those children with diarrhea at admission and 26 (40.6\%) from those without) were given ready-to-use-therapeutic food (RUTF) (Table 3). Presence or absence of diarrhea at admission did not appear to significantly influence the types of therapeutic feeding provided in this study.

\section{Treatment Outcomes}

The SPHERE project standards for an outcome measure of children hospitalised with SAM was employed. Accordingly, a recovery rate of $>75 \%$, a defaulter rate of $<15 \%$, a case fatality rate of $<10 \%$, an average weight gain
Table I Sociodemographic Characteristics and Anthropometric and Laboratory Measurement Results Among Children with SAM Admitted to Jimma University Medical Center, 2018, N= I33

\begin{tabular}{|c|c|c|c|}
\hline \multicolumn{2}{|c|}{$\begin{array}{l}\text { Characteristics or } \\
\text { Measurements }\end{array}$} & \multirow{2}{*}{$\begin{array}{l}\text { Children with } \\
\text { Diarrhea } \\
\text { (n=69) }\end{array}$} & \multirow{2}{*}{$\begin{array}{l}\text { Children } \\
\text { Without } \\
\text { Diarrhea }(n=64) \\
19(29.7 \%) \\
17(26.6 \%) \\
14(21.9 \%) \\
10(15.6 \%) \\
4(6.25 \%)\end{array}$} \\
\hline $\begin{array}{l}\text { Age (in } \\
\text { months) }\end{array}$ & $\begin{array}{l}6-11 \\
12-23 \\
24-35 \\
36-47 \\
48-59\end{array}$ & & \\
\hline Residence & $\begin{array}{l}\text { Rural } \\
\text { Urban }\end{array}$ & $\begin{array}{l}56(81.2 \%) \\
13(18.8 \%)\end{array}$ & $\begin{array}{l}56(87.5 \%) \\
8(12.5 \%)\end{array}$ \\
\hline Sex & $\begin{array}{l}\text { Male } \\
\text { Female }\end{array}$ & $\begin{array}{l}34(49.3 \%) \\
35(50.7 \%)\end{array}$ & $\begin{array}{l}31 \text { (48.4\%) } \\
33(51.6 \%)\end{array}$ \\
\hline MUAC & $\begin{array}{l}<11.5 \mathrm{~cm} \\
>11.5 \mathrm{~cm}\end{array}$ & $\begin{array}{l}59(85.5 \%) \\
10(14.5 \%)\end{array}$ & $\begin{array}{l}48(75.0 \%) \\
16(25.0 \%)\end{array}$ \\
\hline WFH & $\begin{array}{l}<-3 \\
Z \text { score } \\
>-3 \\
Z \text { score }\end{array}$ & $\begin{array}{l}29(42.03 \%) \\
40(58.0 \%)\end{array}$ & $\begin{array}{l}27(42.2 \%) \\
37(57.8 \%)\end{array}$ \\
\hline $\begin{array}{l}\text { Nutritional } \\
\text { diagnoses }\end{array}$ & $\begin{array}{l}\text { Edematous } \\
\text { Non- } \\
\text { edematous }\end{array}$ & $\begin{array}{l}33(47.83 \%) \\
36(52.17 \%)\end{array}$ & $\begin{array}{l}23(35.9 \%) \\
41(64.1 \%)\end{array}$ \\
\hline $\begin{array}{l}\text { Baseline } \\
\text { breast feeding } \\
\text { status }\end{array}$ & $\begin{array}{l}\text { Yes } \\
\text { No }\end{array}$ & $\begin{array}{l}35(50.7 \%) \\
34(49.3 \%)\end{array}$ & $\begin{array}{l}33(51.6 \%) \\
31(48.4 \%)\end{array}$ \\
\hline $\begin{array}{l}\text { Complete } \\
\text { blood count } \\
(\mathrm{CBC})\end{array}$ & $\begin{array}{l}\text { Yes } \\
\text { Not done }\end{array}$ & $\begin{array}{l}59(60.9 \%) \\
10(39.1 \%)\end{array}$ & $\begin{array}{l}40(73.4 \%) \\
24(26.6 \%)\end{array}$ \\
\hline $\begin{array}{l}\text { Hemoglobin } \\
\text { level }(g / d l)\end{array}$ & $\begin{array}{l}<5 \\
5.1-7.0 \\
7.1-8.0 \\
8.1-10.0 \\
>10.0 \\
\text { Unknown }\end{array}$ & $\begin{array}{l}3(4.3 \%) \\
6(8.7 \%) \\
10(14.5 \%) \\
8(11.6 \%) \\
32(46.4 \%) \\
10(14.5 \%)\end{array}$ & $\begin{array}{l}\text { I (I.5\%) } \\
7 \text { (II.0\%) } \\
7 \text { (II.0\%) } \\
\text { II (I7.2\%) } \\
14(21.8 \%) \\
24(37.5 \%)\end{array}$ \\
\hline $\begin{array}{l}\text { Gene-Xpert } \\
\text { /RIF }\end{array}$ & $\begin{array}{l}\text { Yes } \\
\text { Not done }\end{array}$ & $\begin{array}{l}16(23.2 \%) \\
53(76.8 \%)\end{array}$ & $\begin{array}{l}\text { II (17.2\%) } \\
53(82.8)\end{array}$ \\
\hline $\begin{array}{l}\text { Stool } \\
\text { examination }\end{array}$ & $\begin{array}{l}\text { Yes } \\
\text { Not done }\end{array}$ & $\begin{array}{l}41(59.4 \%) \\
28(40.6 \%)\end{array}$ & $\begin{array}{l}30(46.9 \%) \\
34(53.1 \%)\end{array}$ \\
\hline Urine analysis & $\begin{array}{l}\text { Yes } \\
\text { Not done }\end{array}$ & $\begin{array}{l}47(68.1 \%) \\
22(31.9 \%)\end{array}$ & $\begin{array}{l}37(57.8 \%) \\
27(42.2 \%)\end{array}$ \\
\hline $\begin{array}{l}\text { Blood film } \\
\text { test }\end{array}$ & $\begin{array}{l}\text { Yes } \\
\text { Not done }\end{array}$ & $\begin{array}{l}20(29.0 \%) \\
49(71.0 \%)\end{array}$ & $\begin{array}{l}14(21.9 \%) \\
50(78.1 \%)\end{array}$ \\
\hline HIV test & $\begin{array}{l}\text { Yes } \\
\text { Not done }\end{array}$ & $\begin{array}{l}27(39.1 \%) \\
42(60.9 \%)\end{array}$ & $\begin{array}{l}26(40.6 \%) \\
38(59.4 \%)\end{array}$ \\
\hline
\end{tabular}

(Continued) 
Table I (Continued).

\begin{tabular}{|l|l|l|l|}
\hline \multicolumn{2}{|l|}{$\begin{array}{l}\text { Characteristics or } \\
\text { Measurements }\end{array}$} & $\begin{array}{l}\text { Children with } \\
\text { Diarrhea } \\
(\mathbf{n}=69)\end{array}$ & $\begin{array}{l}\text { Children } \\
\text { Without } \\
\text { Diarrhea (n=64) }\end{array}$ \\
\hline $\begin{array}{l}\text { Blood glucose } \\
\text { test }\end{array}$ & $\begin{array}{l}\text { Yes } \\
\text { Not done }\end{array}$ & $\begin{array}{l}18(26.1 \%) \\
51(73.9 \%)\end{array}$ & $\begin{array}{l}19(29.7 \%) \\
45(70.3 \%)\end{array}$ \\
\hline $\begin{array}{l}\text { Blood glucose } \\
\text { level (mg/d) }\end{array}$ & $<54$ & $3(2.9 \%)$ & $2(3.1 \%)$ \\
& $>54$ & $16(23.2 \%)$ & $17(26.6 \%)$ \\
\cline { 2 - 5 } & Unknown & $51(73.9 \%)$ & $45(70.3 \%)$ \\
\hline
\end{tabular}

Abbreviation: HIV, human immunodeficiency virus.

of $\geq 8 \mathrm{~g} / \mathrm{kg} /$ day and an average duration for hospital stay of $<4$ weeks are the acceptable standards. Out of the 133 children included, $25.6 \%$ recovered or achieved nutritional cure, $7.6 \%$ defaulted and $3.8 \%$ died during the data collection period. The overall average weight gain for the children who achieved nutritional cure was $6.9 \mathrm{~g} / \mathrm{kg} / \mathrm{day}$ (Table 4). The overall average weight gain during rehabilitation phase for recovered children $(\mathrm{n}=34)$ was $5 \mathrm{~g} / \mathrm{kg} /$ day (poor) in $16(47.1 \%)$ children, 5 to $10 \mathrm{~g} / \mathrm{kg} /$ day (moderate) and $>10 \mathrm{~g} / \mathrm{kg} /$ day (good) in $10(29.4 \%)$ and $8(23.5 \%)$ children, respectively.

\section{Nutritional Recovery and Survival Analysis}

The overall incidence rate of recovery was 4.2 (95\% CI: 5.2-3.2) per 40 person-days of observation. The incidence rate of recovery was 4.7 (95\% CI: 6.2-3.2) for children who had no diarrhea at admission, and it was slightly slower for children who had diarrhea at admission 3.8 (95\% CI: $5.1-2.5$ ). The overall median time to recovery was 26.0 days (95\%, CI: 23.3-29.70). The median time to recovery for children who had diarrhea at admission was comparable 25.90 days (95\%, CI: 21.90-30.10) to children who had no diarrhea at admission 26.10 days $(95 \% \mathrm{CI}$ : 22.5-29.5). The Kaplan-Meier survival curve indicated that there was no statistically significant difference in the median time to recovery between children who presented with diarrhea at admission and those who were not ( $\log$ rank $=0.017, \mathrm{p}=0.898$ ) (Figure 1).

On the other hand, there was a significant difference ( $\log$ rank $=5.5, \mathrm{p}=0.019)$ in the median nutritional recovery time between children who had comorbid tuberculosis (30.0 days 95\% CI: 27.0-33.0) and those who had not tuberculosis (24.0 days, 95\% CI: 22.0-26-0). Similarly, the median time to nutritional recovery was significantly different (Log rank-
Table 2 Comorbid Medical Conditions Among Children with SAM Admitted to Jimma University Medical Center, 2018, N= 133

\begin{tabular}{|c|c|c|c|}
\hline $\begin{array}{l}\text { Comorbid } \\
\text { Medical } \\
\text { Conditions }\end{array}$ & & $\begin{array}{l}\text { Children with } \\
\text { Diarrhea }(\mathrm{N}=69)\end{array}$ & $\begin{array}{l}\text { Children Without } \\
\text { Diarrhea }(\mathrm{N}=64)\end{array}$ \\
\hline Hypoglycemia & $\begin{array}{l}\text { Yes } \\
\text { No }\end{array}$ & $\begin{array}{l}3(4.35 \%) \\
66(95.65 \%)\end{array}$ & $\begin{array}{l}2(3.1 \%) \\
62(96.9 \%)\end{array}$ \\
\hline $\begin{array}{l}\text { Nutritional } \\
\text { edema }\end{array}$ & $\begin{array}{l}\text { Yes } \\
\text { No }\end{array}$ & $\begin{array}{l}33(47.8 \%) \\
36(52.2 \%)\end{array}$ & $\begin{array}{l}23(35.9 \%) \\
4 I(64.1 \%)\end{array}$ \\
\hline $\begin{array}{l}\text { Kwash- } \\
\text { dermatosis }\end{array}$ & $\begin{array}{l}\text { Yes } \\
\text { No }\end{array}$ & $\begin{array}{l}14(20.3 \%) \\
55(79.7 \%)\end{array}$ & $\begin{array}{l}9(14.0 \%) \\
55(86.0 \%)\end{array}$ \\
\hline Pneumonia & $\begin{array}{l}\text { Yes } \\
\text { No }\end{array}$ & $\begin{array}{l}4(5.8 \%) \\
65(94.2 \%)\end{array}$ & $\begin{array}{l}8(12.5 \%) \\
56(87.5 \%)\end{array}$ \\
\hline Tuberculosis & $\begin{array}{l}\text { Yes } \\
\text { No }\end{array}$ & $\begin{array}{l}12(17.4 \%) \\
57(82.6 \%)\end{array}$ & $\begin{array}{l}12(18.75 \%) \\
52(81.25 \%)\end{array}$ \\
\hline Sepsis & $\begin{array}{l}\text { Yes } \\
\text { No }\end{array}$ & $\begin{array}{l}\text { I (I.45\%) } \\
68 \text { (98.55\%) }\end{array}$ & $\begin{array}{l}2(3.1 \%) \\
62(96.9 \%)\end{array}$ \\
\hline HIV & $\begin{array}{l}\text { Yes } \\
\text { No }\end{array}$ & $\begin{array}{l}2(2.9 \%) \\
67(97.1 \%)\end{array}$ & $\begin{array}{l}\text { I (1.6\%) } \\
63(98.4 \%)\end{array}$ \\
\hline Otitis media & $\begin{array}{l}\text { Yes } \\
\text { No }\end{array}$ & $\begin{array}{l}4(5.8 \%) \\
65(94.2 \%)\end{array}$ & $\begin{array}{l}2(3.1 \%) \\
62(96.9 \%)\end{array}$ \\
\hline $\begin{array}{l}\text { Urinary tract } \\
\text { Infections }\end{array}$ & $\begin{array}{l}\text { Yes } \\
\text { No }\end{array}$ & $\begin{array}{l}4(5.8 \%) \\
65(94.2 \%)\end{array}$ & $\begin{array}{l}4(6.25 \%) \\
60(93.75 \%)\end{array}$ \\
\hline Rickets & $\begin{array}{l}\text { Yes } \\
\text { No }\end{array}$ & $\begin{array}{l}7(10.15 \%) \\
62(89.85 \%)\end{array}$ & $\begin{array}{l}10(15.63 \%) \\
54(84.37 \%)\end{array}$ \\
\hline Oral thrush & $\begin{array}{l}\text { Yes } \\
\text { No }\end{array}$ & $\begin{array}{l}10(14.5 \%) \\
59(85.5 \%)\end{array}$ & $\begin{array}{l}5(7.8 \%) \\
59(92.2 \%)\end{array}$ \\
\hline Anemia & $\begin{array}{l}\text { Yes } \\
\text { No }\end{array}$ & $\begin{array}{l}27(39.13 \%) \\
42(60.87 \%)\end{array}$ & $\begin{array}{l}26(40.6 \%) \\
38(59.4 \%)\end{array}$ \\
\hline Malaria & $\begin{array}{l}\text { Yes } \\
\text { No }\end{array}$ & $\begin{array}{l}3(4.35 \%) \\
66(95.65 \%)\end{array}$ & $\begin{array}{l}0(0 \%) \\
64(100 \%)\end{array}$ \\
\hline
\end{tabular}

5.4, $\mathrm{p}=0.020$ ) between children who had comorbid anemia (30 days, 95\% CI: 25.0-30.0) and children who had no anemia (23.0 days, 95\% CI: 19.5-26.5) (Table 5).

\section{Factors Associated with Nutritional Recovery}

In the bivariate regression analysis, presence of edema, pneumonia, tuberculosis, Kwash-dermatosis, feeding method, vitamin A therapy, length of stabilization phase, treatment failure and inpatient complications were significantly associated with the nutritional recovery of children. However, multivariate analyses showed that only three factors (presence of treatment failure, tuberculosis, and 
Table 3 Medication and Therapeutic Feeding Among Children with SAM Admitted to Jimma University Medical Center, 2018, $\mathrm{N}=133$

\begin{tabular}{|c|c|c|c|}
\hline \multicolumn{2}{|c|}{$\begin{array}{l}\text { Medication and } \\
\text { Feeding Given }\end{array}$} & \multirow{2}{*}{$\begin{array}{l}\begin{array}{l}\text { Children with } \\
\text { Diarrhea }(\mathrm{N}=69)\end{array} \\
69(100 \%)\end{array}$} & \multirow{2}{*}{$\begin{array}{l}\text { Children Without } \\
\text { Diarrhea }(\mathbf{N}=64)\end{array}$} \\
\hline F-75 & Yes & & \\
\hline$F-100$ & $\begin{array}{l}\text { Yes } \\
\text { No }\end{array}$ & $\begin{array}{l}60(87.0 \%) \\
9(13.0 \%)\end{array}$ & $\begin{array}{l}55(85.9 \%) \\
9(14.1 \%)\end{array}$ \\
\hline RUTF & $\begin{array}{l}\text { Yes } \\
\text { No }\end{array}$ & $\begin{array}{l}27(39.13 \%) \\
42(60.87 \%)\end{array}$ & $\begin{array}{l}26(40.6 \%) \\
38(59.4 \%)\end{array}$ \\
\hline Vitamin A & $\begin{array}{l}\text { Yes } \\
\text { No }\end{array}$ & $\begin{array}{l}38(55.1 \%) \\
31(44.9 \%)\end{array}$ & $\begin{array}{l}42(65.6 \%) \\
22(34.4 \%)\end{array}$ \\
\hline Folic Acid & Yes & $69(100 \%)$ & $64(100 \%)$ \\
\hline $\begin{array}{l}\text { Oral } \\
\text { antibiotics }\end{array}$ & $\begin{array}{l}\text { Yes } \\
\text { No }\end{array}$ & $\begin{array}{l}7(10.15 \%) \\
62(89.85 \%)\end{array}$ & $\begin{array}{l}25(39.1 \%) \\
39(60.9 \%)\end{array}$ \\
\hline $\begin{array}{l}\text { Parenteral } \\
\text { Antibiotics }\end{array}$ & $\begin{array}{l}\text { Yes } \\
\text { No }\end{array}$ & $\begin{array}{l}62(89.85 \%) \\
7(10.15 \%)\end{array}$ & $\begin{array}{l}40(62.5 \%) \\
24(37.5 \%)\end{array}$ \\
\hline Iron & $\begin{array}{l}\text { Yes } \\
\text { No }\end{array}$ & $\begin{array}{l}28(40.6 \%) \\
4 I(59.4 \%)\end{array}$ & $\begin{array}{l}25(39.1 \%) \\
39(60.9 \%)\end{array}$ \\
\hline Mebendazole & $\begin{array}{l}\text { Yes } \\
\text { No }\end{array}$ & $\begin{array}{l}41(59.4 \%) \\
28(40.6 \%)\end{array}$ & $\begin{array}{l}38(59.4 \%) \\
26(40.6 \%)\end{array}$ \\
\hline ReSoMal & $\begin{array}{l}\text { Yes } \\
\text { No }\end{array}$ & $\begin{array}{l}59(85.5 \%) \\
10(14.5 \%)\end{array}$ & $\begin{array}{l}4(6.25 \%) \\
60(93.75 \%)\end{array}$ \\
\hline IV fluid & $\begin{array}{l}\text { Yes } \\
\text { No }\end{array}$ & $\begin{array}{l}3(4.35 \%) \\
66(95.65 \%)\end{array}$ & $\begin{array}{l}\text { I (1.56\%) } \\
63(98.44 \%)\end{array}$ \\
\hline $\begin{array}{l}\text { Blood } \\
\text { Transfusion }\end{array}$ & $\begin{array}{l}\text { Yes } \\
\text { No }\end{array}$ & $\begin{array}{l}3(4.35 \%) \\
66(95.65 \%)\end{array}$ & $\begin{array}{l}\text { I (I.56\%) } \\
63 \text { (98.44\%) }\end{array}$ \\
\hline
\end{tabular}

Abbreviations: F-75, formula-75; F-I00, formula 100; ReSoMal, rehydration solution for malnutrition; RUTF, ready to use therapeutic food; IV, intravenous.

anemia) were associated with nutritional recovery. Thus, in children with comorbid tuberculosis, the chance of recovery from SAM was $80.7 \%$ lower compared with children without tuberculosis (adjusted hazard ratio $(\mathrm{AHR})=0.193,95 \% \mathrm{CI}: 0.06-0.62 ; \mathrm{P}=0.006)$. Likewise, children with comorbid anemia had $68.2 \%$ lower probability of recovery from SAM compared with children without anemia (AHR $=0.322 ; 95 \%$ CI: 0.139-0.743; $\mathrm{P}=0.008$ ). Besides, children who experienced treatment failure during admission had $83.5 \%$ lower probability to recover from SAM compared with those children with no treatment failure during hospitalisation $(\mathrm{AHR}=0.165 ; 95 \%$ CI: 0.164-0.806; $\mathrm{P}=0.008$ ) (Table 6).

\section{Discussion}

In this study, case fatality rate was $3.8 \%$ which was within the national and international standards of below $10 \% .{ }^{11}$ Similarly, the defaulting rate $(7.6 \%)$ was found to be within the acceptable range of national and international recommendations which is $<15.0 \% .{ }^{11}$ However, the recovery rate $(25.6 \%)$ was much lower than both national and international minimum acceptable value ( $>75 \%$ ) for inpatient management of SAM. ${ }^{11}$ This is comparable with the findings of study conducted in Latin America $(61.7 \%)^{12}$ and some African countries (53.7\%). ${ }^{13}$ On the contrary, the recovery rate in our study was higher than reports from studies conducted in some African countries. ${ }^{14}$ The higher recovery rate in our study might be due to delayed transition to the rehabilitation phase (catch-up feeding), failure to increase caloric intake during the rehabilitation phase or preference for outpatient nutritional rehabilitation.

The overall median time to recovery (26.0 days) in the present study was comparable with the international recommendation which is less than four weeks for inpatient management of SAM. ${ }^{11}$ Compared with our findings, studies conducted in Bangladesh ${ }^{15}$ and India ${ }^{16}$ reported shorter median time to recovery (less than 3 weeks versus

Table 4 Performance Indicators versus Treatment Outcomes for Children with SAM Admitted to Jimma University Medical Center, $2018, N=133$

\begin{tabular}{|c|c|c|c|c|c|c|}
\hline \multirow{2}{*}{\multicolumn{2}{|c|}{ Category }} & \multicolumn{5}{|c|}{ Performance Indicators } \\
\hline & & $\begin{array}{l}\text { Recovery } \\
\text { Rate }\end{array}$ & $\begin{array}{l}\text { Defaulter } \\
\text { Rate }\end{array}$ & $\begin{array}{l}\text { Death } \\
\text { Rate }\end{array}$ & $\begin{array}{l}\text { Average Length of Hospital } \\
\text { Stays }\end{array}$ & $\begin{array}{l}\text { Average Weight } \\
\text { Gain }\end{array}$ \\
\hline \multirow{3}{*}{\multicolumn{2}{|c|}{$\begin{array}{l}\text { Children with diarrhea } \\
\text { Children without diarrhea } \\
\text { Overall }\end{array}$}} & 18 (52.9\%) & $5(50.0 \%)$ & $4(80.0 \%)$ & 25.9 days & $6.3 \mathrm{~g} / \mathrm{kg} / \mathrm{day}$ \\
\hline & & $16(47.1 \%)$ & $5(50.0 \%)$ & I (25.0\%) & 26.1 days & $7.6 \mathrm{~g} / \mathrm{kg} / \mathrm{day}$ \\
\hline & & $25.6 \%$ & $7.6 \%$ & $3.8 \%$ & 26 days & $6.9 \mathrm{~g} / \mathrm{kg} /$ day \\
\hline SPHERE* & Acceptable & $>75 \%$ & $<15 \%$ & $<10 \%$ & $<4$ weeks & $\geq 8 \mathrm{~g} / \mathrm{kg} /$ day \\
\hline Standards & Alarming & $<50 \%$ & $>25 \%$ & $>15 \%$ & $>6$ weeks & $\leq 8 \mathrm{~g} / \mathrm{kg} /$ day \\
\hline
\end{tabular}

Note: *The sphere project. 


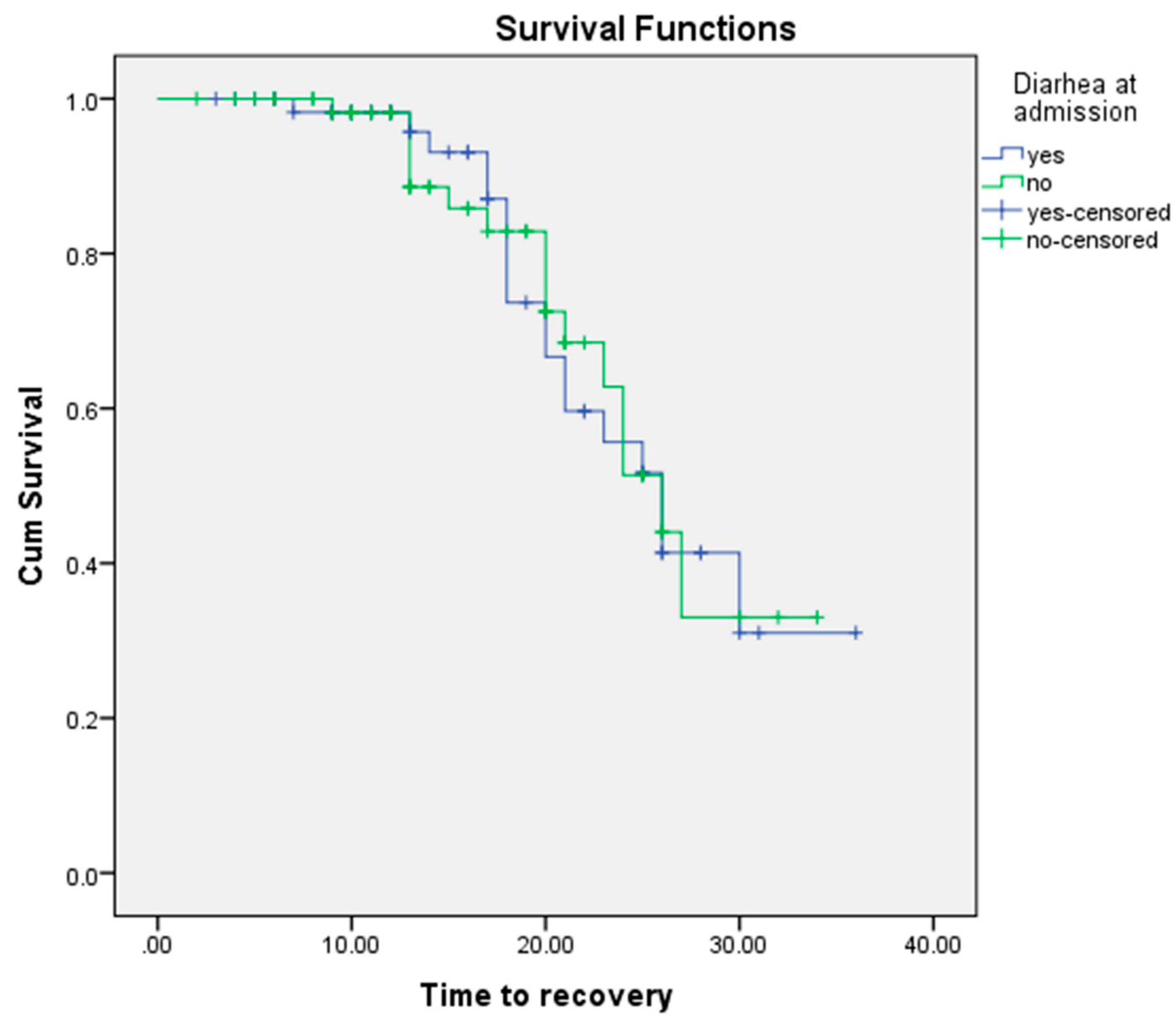

Figure I Kaplan-Meier estimate of recovery time in children with severe acute malnutrition by status of diarrhea admitted to Jimma University Medical Center, 20 I8, N= I33.

15.5 days). On the contrary, in a study conducted in Malawi ${ }^{17}$ longer median time to recovery (49.0 days) has been reported. The discrepancies in the findings could be due to the variation in the quality of care at the health-care settings, types of medical comorbidities and complications during hospitalisation.
The overall average daily weight gain in the present study was slightly lower than the international recommendation $(6.9 \mathrm{~g} / \mathrm{kg} / \text { day versus } \geq 8 \mathrm{~g} / \mathrm{kg} / \mathrm{day})^{11}$ and the findings of studies conducted in some Asian countries $(9.3 \mathrm{~g} / \mathrm{kg} / \mathrm{day}) .{ }^{18}$ However, it is slightly higher compared with reports from some African countries $(5.6 \mathrm{~g} / \mathrm{kg} /$ day $) .{ }^{14}$ The variations in the

Table 5 Recovery Time of Severe Acute Malnutrition by Characteristics of Children Admitted to Jimma University Medical Center, $2018, N=133$

\begin{tabular}{|c|c|c|c|c|c|c|}
\hline \multicolumn{2}{|c|}{$\begin{array}{l}\text { Medical and Treatment } \\
\text { Statuses of Children }\end{array}$} & $\begin{array}{l}\text { Children with } \\
\text { Diarrhea }(n=69)\end{array}$ & $\begin{array}{l}\text { Children Without } \\
\text { Diarrhea }(n=64)\end{array}$ & $\begin{array}{l}\text { Median Time to Recovery } \\
\text { Estimate }(95 \% \mathrm{Cl})\end{array}$ & $\begin{array}{l}\text { Log Rank } \\
\text { Value }\end{array}$ & P-values \\
\hline Tuberculosis & $\begin{array}{l}\text { No } \\
\text { Yes }\end{array}$ & $\begin{array}{l}57 \\
12\end{array}$ & $\begin{array}{l}52 \\
12\end{array}$ & $\begin{array}{l}24.0(22.0-26-0) \\
30.0(27.0-33.0)\end{array}$ & 5.5 & 0.019 \\
\hline Anemia & $\begin{array}{l}\text { No } \\
\text { Yes }\end{array}$ & $\begin{array}{l}42 \\
27\end{array}$ & $\begin{array}{l}38 \\
26\end{array}$ & $\begin{array}{l}23.0(19.5-26.5) \\
30.0(25.0-30.0)\end{array}$ & 5.4 & 0.020 \\
\hline $\begin{array}{l}\text { Kwash- } \\
\text { dermatosis }\end{array}$ & $\begin{array}{l}\text { No } \\
\text { Yes }\end{array}$ & $\begin{array}{l}55 \\
14\end{array}$ & $\begin{array}{l}55 \\
9\end{array}$ & $\begin{array}{l}18.0(16.0-20.0) \\
24.0(14.0-34.0)\end{array}$ & 4.67 & 0.031 \\
\hline $\begin{array}{l}\text { Treatment } \\
\text { failure }\end{array}$ & $\begin{array}{l}\text { No } \\
\text { Yes }\end{array}$ & $\begin{array}{l}59 \\
10\end{array}$ & $\begin{array}{l}55 \\
9\end{array}$ & $\begin{array}{l}23.0(21.0-25.0) \\
33.0(30.0-37.0)\end{array}$ & 11.6 & 0.001 \\
\hline
\end{tabular}


Table 6 Predictors of Nutritional Recovery in Children with Severe Acute Malnutrition Admitted to Jimma University Medical Center, $2018, N=133$

\begin{tabular}{|c|c|c|c|c|c|}
\hline Factors & & $\begin{array}{l}\text { Children with } \\
\text { Diarrhea }(N=69)\end{array}$ & $\begin{array}{l}\text { Children Without } \\
\text { Diarrhea }(\mathrm{N}=64)\end{array}$ & $\operatorname{AHR}(95 \%, \mathrm{Cl})$ & P-values \\
\hline Residence & $\begin{array}{l}\text { Rural } \\
\text { Urban }\end{array}$ & $\begin{array}{l}56 \\
13\end{array}$ & $\begin{array}{l}56 \\
8\end{array}$ & $\begin{array}{l}\text { I } \\
\text { I.0I (0.34-2.97) }\end{array}$ & 0.992 \\
\hline Edema & $\begin{array}{l}\text { No } \\
\text { Yes }\end{array}$ & $\begin{array}{l}36 \\
33\end{array}$ & $\begin{array}{l}41 \\
23\end{array}$ & $\begin{array}{l}\text { I } \\
3.70(0.42-3 I .4 I)\end{array}$ & 0.231 \\
\hline Vomiting at admission & $\begin{array}{l}\text { No } \\
\text { Yes }\end{array}$ & $\begin{array}{l}29 \\
40\end{array}$ & $\begin{array}{l}54 \\
10\end{array}$ & $\begin{array}{l}\text { I } \\
0.36(0.16-0.81)\end{array}$ & 0.053 \\
\hline Pneumonia & $\begin{array}{l}\text { No } \\
\text { Yes }\end{array}$ & $\begin{array}{l}65 \\
4\end{array}$ & $\begin{array}{l}56 \\
8\end{array}$ & $\begin{array}{l}\text { I } \\
0.46(0.14-1.55)\end{array}$ & 0.210 \\
\hline Vitamin A & $\begin{array}{l}\text { No } \\
\text { Yes }\end{array}$ & $\begin{array}{l}31 \\
38\end{array}$ & $\begin{array}{l}22 \\
42\end{array}$ & $\begin{array}{l}\text { I } \\
1.69(0.73-3.88)\end{array}$ & 0.220 \\
\hline Anemia & $\begin{array}{l}\text { No } \\
\text { Yes }\end{array}$ & $\begin{array}{l}42 \\
27\end{array}$ & $\begin{array}{l}38 \\
26\end{array}$ & $\begin{array}{l}\text { I } \\
0.32(0.14-0.74)\end{array}$ & $0.008^{*}$ \\
\hline Kwash-dermatosis & $\begin{array}{l}\text { No } \\
\text { Yes }\end{array}$ & $\begin{array}{l}55 \\
14\end{array}$ & $\begin{array}{l}55 \\
9\end{array}$ & $\begin{array}{l}\text { I } \\
0.75(0.19-2.97)\end{array}$ & 0.681 \\
\hline Tuberculosis & $\begin{array}{l}\text { No } \\
\text { Yes }\end{array}$ & $\begin{array}{l}57 \\
12\end{array}$ & $\begin{array}{l}52 \\
12\end{array}$ & $\begin{array}{l}\text { I } \\
0.19(0.06-0.62)\end{array}$ & $0.006 *$ \\
\hline Feeding method & $\begin{array}{l}\text { Oral } \\
\text { NGT }\end{array}$ & $\begin{array}{l}46 \\
23\end{array}$ & $\begin{array}{l}44 \\
20\end{array}$ & $\begin{array}{l}\text { I } \\
0.62(0.22-\mid .8 I)\end{array}$ & 0.384 \\
\hline Inpatient complication & $\begin{array}{l}\text { No } \\
\text { Yes }\end{array}$ & $\begin{array}{l}51 \\
18\end{array}$ & $\begin{array}{l}54 \\
10\end{array}$ & $\begin{array}{l}\text { I } \\
0.41(0.16-1.03)\end{array}$ & 0.057 \\
\hline Treatment failure & $\begin{array}{l}\text { No } \\
\text { Yes }\end{array}$ & $\begin{array}{l}59 \\
10\end{array}$ & $\begin{array}{l}55 \\
9\end{array}$ & $\begin{array}{l}\text { I } \\
0.17(0.16-0.8 I)\end{array}$ & $0.026^{*}$ \\
\hline Length of stabilisation phase & $\begin{array}{l}<7 \text { days } \\
\geq 7 \text { days }\end{array}$ & $\begin{array}{l}25 \\
44\end{array}$ & $\begin{array}{l}23 \\
41\end{array}$ & $\begin{array}{l}\text { I } \\
0.48(0.23-1.02)\end{array}$ & 0.055 \\
\hline
\end{tabular}

Note: *Statistically significant.

Abbreviations: AHR, adjusted hazard ratio; $\mathrm{Cl}$, confidence interval; NGT, nasogastric tube.

findings could be due to inappropriate feeding, inpatient complication, and sub-optimal average daily weight gain.

In this study, it was found that the presence of tuberculosis was an independent predictor of time to recover and thus, children with tuberculosis had lower probability of recovery from SAM. In line with our finding, the presence of tuberculosis comorbidity was reported as an independent predictor of mortality in a study conducted in Bangladesh. ${ }^{15}$ It is known that tuberculosis worsens undernutrition as patients with active tuberculosis are often in a catabolic state, and experience weight loss from decreased dietary intake as a result of loss of appetite, nausea and abdominal pain.

Likewise, in this study, we found that the probability of recovery from SAM was $68.8 \%$ lower in children with anemia compared with children without anemia. Contrary to our findings, a study conducted in Burkina Faso reported that presence of anemia has no impact on recovery rate in children with SAM. ${ }^{19}$ The high prevalence of anemia (83.3\%) in the study population might have limited the above study ${ }^{19}$ to detect meaningful differences in the recovery rate between children with anemia and those children without anemia. The present study also found that treatment failure is an independent predictor of time to recovery. Children who experienced treatment failure during admission were found to have $83.5 \%$ lower probability of recovery from SAM when compared with children who did not experience treatment failure.

This study is the first longitudinal study to identify factors associated with treatment outcomes in children 
severe acute malnutrition in Ethiopia. However, it is not without limitation. The small sample size and being limited to a single medical center may limit generalizability of the findings of this study.

\section{Conclusion}

The nutritional recovery rate and average daily weight gain were found to be suboptimal in the study population. However, the median time to nutritional recovery was within the recommended national standard. Children who had diarrhea at admission did not have lower recovery rate and prolonged time to recovery compared with children who had no diarrhea at admission. Optimal daily weight gain and presence of comorbidities such as tuberculosis and anemia contributed to prolonged stabilization phase and time to achieve nutritional cure. Optimising the average daily weight gain and clinical management of comorbidities may enhance nutritional recovery in hospitalised children with SAM.

\section{Acknowledgments}

The authors acknowledge participants of the study for their time, and Jimma University for funding this study.

\section{Author Contributions}

All authors contributed to data analysis, drafting or revising the article, gave final approval of the version to be published, and agree to be accountable for all aspects of the work.

\section{Disclosure}

The authors declare that they have no competing interests.

\section{References}

1. Black RE, Victora CG, Walker SP, et al. Maternal and Child Nutrition 1 Maternal and child undernutrition and overweight in low-income and middle-income countries. lancet. 2013;6736(13):427-451.
2. United Nation children's Funds, World Health Organization and the WBG. Levels and Trends in Child Malnutrition; 2017.

3. Wakai S, Ito N, Adachi N, Ueda D, Tsutsumi H, Chiba S. Hospice care for children [8]. Lancet. 1996;348(9034):1102. doi:10.1016/ S0140-6736(05)64448-0

4. Uinted Nation Children's Fund(UNICEF). Management of Severe Acute Malnutrition in Children: Working Towards Results at Scale; 2015.

5. Federal Ministry of Health(FMOH) Ethiopia. Protocol for the Management of Severe Acute Malnutrition; 2007:1-122

6. Central Statistical Agency (CSA), Ababa A. Ethiopia Mini Demographic and Health Survey. Ethiopia Demographic and Health Survey; 2014.

7. Central Statistical Agency (CSA) Addis Ababa E. Ethiopia Demographic and Health Survey; 2016.

8. United Nation High Commission For Refugees(UNHCR), World Food Programme (WFP) and WHO (WHO). Guidelines for Selective Feeding: The Management of Malnutrition in Emergencies; 2011.

9. World Health Organization (WHO). WHO Child Growth Standards. Dev Med Child Neurol. 2009;51(12):1002. doi:10.1111/j.14698749.2009.03503.x

10. Ethiopian Federal ministry of health. Guidelines for the Management of Acute Malnutrition; 2016.

11. The Sphere Project. Humanitarian Charter and Minimum Standards in Disaster Response; 2011.

12. Bernal ÃC, Vela C, Alcaraz G, Botero ÃJ. Treatment of severe malnutrition in children: experience in implementing the World Health Organization Guidelines in Turbo, Colombia. J Pediatr Gastroenterol Nutr. 2008;46:322-328.

13. Irena AH, Mwambazi M, Mulenga V. Diarrhea is a major killer of children with severe acute malnutrition admitted to inpatient set-up in Lusaka, Zambia. Nutr J. 2011;10(1):110. doi:10.1186/1475-2891-10110

14. John FK. Clinical outcomes of children aged 6 to 59 months with severe acute malnutrition admitted to mbagathi district hospital department of paediatrics \& child health. Arch Dis Child. 2013;94:512-516.

15. Hossain I, Dodd NS, Ahmed T, et al. Experience in managing severe malnutrition in a government tertiary treatment facility in Bangladesh. J Health Popul Nutr. 2009;27(1):72.

16. Shah RH, Javdekar BB, Rh S, Contemp IJ, May P. Management of children with severe acute malnutrition: experience of nutrition rehabilitation centre at Baroda, Gujarat. Indian Pediatr. 2014;1(1):3-6.

17. Sadler K, Kerac M, Collins S, Khengere H, Nesbitt A. Improving the Management of severe acute malnutrition in an area of high HIV prevalence. J Trop Pediatr. 2018;54(6):364-369.

18. Singh P, Kumar P, Rohatgi S, Basu S, Aneja S. Experience and outcome of children with severe acute malnutrition using locally prepared therapeutic diet. Indian J Pediatr. 2016;83:3-8.

19. Savadogo LGB, Zoetaba I, Ilboudo B, Kinda M. Impact of anemia on mortality and nutritional recovery among hospitalized severely malnourished children in Burkina Faso. Open J Pediatr. 2014;115-120.

Pediatric Health, Medicine and Therapeutics

Dovepress

\section{Publish your work in this journal}

Pediatric Health, Medicine and Therapeutics is an international, peerreviewed, open access journal publishing original research, reports, editorials, reviews and commentaries. All aspects of health maintenance, preventative measures and disease treatment interventions are addressed within the journal. Practitioners from all disciplines are invited to submit their work as well as healthcare researchers and patient support groups. The manuscript management system is completely online and includes a very quick and fair peer-review system. Visit http://www.dovepress.com/testimonials.php to read real quotes from published authors. 